I OF I 


\title{
Radiation Damage in the SDC Hadronic Endcap Calorimeter
}

\author{
Dan Green \\ Fermi National Accelerator Laboratory \\ P.O. Box 500, Batavia, Illinois 60510
}

December 1992
PECEIVED
FEB -81993
OSTI




\section{Disclaimer}

This report was prepared as an account of work sponsored by an agency of the United States Government. Neither the United States Government nor any agency thereof, nor any of their employees, makes any warranty, express or implied, or assumes any legal liability or responsibility for the accuracy, completeness, or usefulness of any information, apparatus, product, or process disclosed, or represents that its use would not infringe privately owned rights. Reference herein to any specific commercial product, process, or service by trade name, trademark, manufacturer, or otherwise, does not necessarily constitute or imply its endorsement, recommendation, or favoring by the United States Government or any agency thereof. The views and opinions of authors expressed herein do not necessarily state or reflect those of the United States Government or any agency thereof. 


\title{
RADIATION DAMAGE IN THE SDC HADRONIC ENDCAP CALORIMETER
}

\author{
Dan Green \\ Fermi National Accelerator Laboratory
}

\section{Introduction}

Detectors for the SSC face a radiation field which is very dependent on angle [1]. For example, the SDC "barrel" calorimeter can function well for 100 year operation of the SSC runnning at design luminosity, while the small angle "forward calorimeter" faces Grad of radiation in the same period [2]. The SDC "endcap" calorimeter is in an intermediate location. One wishes to examine whether it might be possible to use conventional scintillator technology with periodic refurbishment in the endcap. The angular range covered by the endcap spans the region, $1.4<\eta<3.0$. In this note, only the hadronic (HAD) compartment is considered. The electromagnetic (EM) compartment is considered elsewhere.

\section{Minbias Energy Deposition - EM and HAD Dose}

The energy deposition in the endcap is assumed to be due to charged and neutral pions from "minbias" events. The typical transverse momentum is, $\langle\mathrm{kt}>\sim 0.7 \mathrm{GeV}$, so that, at $\eta=3.0$, the typical pion energy is $7 \mathrm{GeV}$. The longitudinal energy profile for these low energy hadrons and neutral pions, as directly measured, has not been used here. Rather, approximate values are obtained using data from a test at Fermilab. The test module consisted of 40 layers of $1 / 8$ " $\mathrm{Pb}$, followed by 55 layers of 1 " Fe. This configuration of the test module, the Hanging File (HF), is quite similar to the SDC central calorimeter design [2].

The lowest reliable energy found in the HF data was $10 \mathrm{GeV}$ [3]. Depth profiles for $10 \mathrm{GeV}$ incident electrons and pions on the HF module are shown in Fig. 1. These profiles have been used to derive the radiation dose throughout the endcap as a function of depth and angle. The electron data was assumed to represent the profile of $10 \mathrm{GeV}$ neutral pions. This assumption ignores the slow logarithmic dependence of the EM longitudinal shower profile on incident energy. 
The dose for neutral pions was taken to be proportional to the energy profile of the electrons. The peak EM dose was taken to be $50 \mathrm{Mrad}$ at $\eta=3$. This dose is appropriate to the maximum endcap dose in 100 year operation at SSC design luminosity [1]. By comparison, the maximum EM dose in the wide angle barrel module ranges from 0.2 Mrad at $\eta=0$ to $0.6 \mathrm{Mrad}$ at $\eta=1.5$.

The dose due to incident hadrons was assumed to be twice that due to charged pions since the number of charged pions is twice that of neutral pions. The resulting neutral dose, and total dose at $\eta=3$ for 100 year operation at SSC design luminosity, is shown in Fig. 2. The neutral dose is well conta ned in the EM compartment, with a peak of $50 \mathrm{Mrad}$. The dose due to charged pions penetrates into the HAD compartment, leading to a peak dose of $\sim 12 \mathrm{Mrad}$. The ratio of EM to HAD dose (50 Mrad/12 Mrad) can be understood roughly as the ratio of radiation length to interaction length taking into account a factor of 2 in charged/neutral incident energy.

The angular dependence of the dose follows from elementary considerations. The solid angle goes as the inverse square of the sine of the polar angle. The energy flow picks up a third power of the sine, implying that the dose scales in angle as the inverse third power of the sine of the polar angle [4]. The dose contours shown in Fig. 3 then follow from the depth profile shown in Fig. 2 and the assumed scaling in angle. Note the steep falloff with $\eta$. The peak dose of $12 \mathrm{Mrad}$ in HAD at $\eta=3$ falls to $<4 \mathrm{Mrad}$ for $\eta<2.5$. The values given here are in reasonable agreement with those computed by slightly different methods [5].

\section{Dose/Damage Relationship}

Given the dose, one needs to relate it to the damage. The damage is taken to be measured by the light output after irradiation relative to the light output before exposure. This quantity is defined to be $1-d$ where $d$ is called the "damage". It has been found experimentally [6] that 1-d is roughly related to the dose, $D$, as;

$$
(1-d)=e^{-D / D o}
$$

In Eq. 1, Do is a characteristic dose defining a damage, $d$, of 63\%. Data [6] relating $d$ and $\mathrm{D}$ are shown in Fig. 4. The basic optics design for tiles (SCSN81) and WLS (BCF91) in a "sigma" [2] layout appropriate to the SDC barrel has a characteristic dose, Do $=3.8 \mathrm{Mrad}$. Given that the 100 year maximum dose in the barrel is $<0.6 \mathrm{Mrad}$, the peak damage in the barrel EM compartment is then $<25 \%$. This damage is known not to induce an unacceptable error in the EM energy measurement [2], [9]. The data with Do of $8.5 \mathrm{Mrad}$ 
corresponds to the same tile and WLS fiber material, but arranged in a "multifiber" layout which reduces the optical path length of the blue light.

We assume that the relationship given in Eq. 1 holds for all depths and not just for the electromagnetic shower maximum. That assumption is in rough agreement with the experimental measurements [6]. The resulting contours of relative light output in depth and $\eta$ are shown in Fig. 5. Note that, if Do $=10 \mathrm{Mrad}$, the contours with light loss $>30 \%$ in 100 year operation cover only a very small region of the HAD compartment. Specifically, small means that the region consists of only 25 layers out of 55 in depth and only the angular region covering $\eta>2.5$. This region corresponds roughly to depths from 1 to 5 absorption lengths and angles from 5.7 to 9.4 degrees.

Note that values of Do $~ 10 \mathrm{Mrad}$ have already been achieved as shown in Fig. 4 [6]. In addition, 3HF scintillator is known to be more radiation resistant than SCSN81. The use of 3HF tile with O2 WLS fiber in a "multifiber" geometry is expected to raise the value of Do to well in excess of $10 \mathrm{Mrad}$. Although the light output is reduced for this tile and WLS combination, it must be remembered that the physics variable is $\mathrm{Pt}$ while the light output is proportional to $\mathrm{P}$. At $\eta=3, \mathrm{P}$ and $\mathrm{Pt}$ differ by an order of magnitude which is greater than the light loss for $3 \mathrm{HF} / \mathrm{O} 2$ with respect to SCSN81/BCF91 (4:1). Preliminary data on 3HF/02 multifiber yields a characteristic dose of $26 \mathrm{Mrad}$ [7].

One can also consider the refurbishment of scintillator as an operating cost. In that case, the question is when does one suffer a light loss which is unacceptable? Assuming $\mathrm{Do}=10 \mathrm{Mrad}$, and that unacceptable loss is $30 \%$, (or $\mathrm{D}=3.6 \mathrm{Mrad}$ ) then the HAD plastic at $\eta=3$ must be repaired every 30 years. Since the SDC silicon devices are more sensitive, the replacement of a small portion of the endcap plastic at small angles is unlikely to be a limitation to SDC operations.

Another possibility is to "remask" the optical depth response [8]. If Do $=25 \mathrm{Mrad}$, then $30 \%$ damage occurs at $D=8.9 \mathrm{Mrad}$. If one can remask once, then a dose of 17.8 Mrad may be tolerated. Therefore, a HAD made of 3HF/02 with multifiber readout and capable of 1 remask would never require scintillator replacement over the 100 year lifetime operation of SDC.

\section{Hanging File Test Data and "Induced Constant Term"}

The relationship of peak damage to calorimeter response has previously been studied using a homogenous Fe calorimeter used in neutrino physics experiments [4]. The "minbias" energy was $15 \mathrm{GeV}$, and the SDC geometry was not well modeled. The present study attempts to be more realistic. 
The damage makes the calorimeter a nonlinear medium. This nonlinearity induces a nonlinearity and an additional error into the calorimeter energy resolution. In Ref. 4, it was estimated that $10 \%$ peak damage induces a $6 \%$ energy nonlinearity. The induced "constant term" was estimated to be $2 \%$ for every $10 \%$ peak damage. The nonlinearity and constant term were observed to be roughly proportional to the peak damage.

Hanging file (HF) data [3] were used in this note in order to give a more accurate representation of the SDC calorimeter. Event by event energy profiles in depth are shown in Fig 6. Clearly, the hadronic showers have very large longitudinal fluctuations. In particular, the neutral content of the shower is seen to fluctuate enormously.

The dose profile shown in Fig. 2 was used for various damage estimates as done in Ref. 4. The relationship given in Eq. 1 was then used to relate dose, $D$, to damage $d$. That damage profile was used to weight the light output from each of the 95 depth segments, $W T(z)=1-d(z)$. The energy sum for the damaged HAD module was then taken to be the sum of the layer contributions $\sum_{i=1}^{95} E i * W T i$. In particular, it was assumed that the EM compartment was periodically replaced, so that $d=0$ for all EM layers, $i=1,40[5]$.

The weighted energy sums for peak $\mathrm{HAD}$ damages of $0.0,0.2,0.4$, and 0.6 are shown in Fig. 7. The two main effects of the damage are to reduce the light output and widen the distribution as $d$ increases. The mean and standard deviation of the distributions for each peak damage are shown in Fig 8. The mean fractional energy shift is shown in Fig. 8a. Clearly, the shift is linearly related to the peak damage. The slope is $5 \%$ shift for each $10 \%$ peak damage, in good agreement with Ref. 4 . Therefore, it should be easy to correct for the mean response using the system of radioactive sources which SDC proposes to use for monitoring the performance of the calorimeter [2].

The fractional energy resolution for $250 \mathrm{GeV}$ pions is shown in Fig. $8 \mathrm{~b}$ as a function of peak HAD damage. Clearly, $<30 \%$ peak damage does not degrade the detector performance significantly. Unfolding in quadrature, the induced constant term is roughly $1.5 \%$ for each $10 \%$ peak HAD damage. This value is in decent agreement with the $2 \%$ per $10 \%$ value found in an homogeneous Fe calorimeter. Since we assumed here that the EM compartment would be refurbished, we expect a slightly softer dependence on peak HAD damage in this study.

One can use Fig. 5 and Fig. 8 to infer the HAD region where the calorimeter performance is unacceptably degraded. Clearly, the region with $30 \%$ peak HAD damage, or $4.5 \%$ induced constant term, is a small fraction of the endcap. In fact, as stated above, there is no repair needed in the HAD compartment of the endcap over the life of the SDC 
detector if plastic with Do $\simeq 25$ Mrad is used and 1 "remasking" [2] of the optical cookie is allowed.

\section{Summary}

The radiation dose profile in depth in the SDC endcap calorimeter HAD compartment was estimated using HF test beam data. The response of the scintillating plastic to that dose was determined using e beam irradiations taken at IHEP/Beijing. The region with relative light output reduced to $<70 \%$ is nonexistent for presently tested plastics after 74 year operation at full SSCL luminosity. After 100 year (lifetime) operation, only a small region of the endcap will ever need to even be remasked. One remasking of the region around $\eta=3$ would insure that the HAD endcap never needed to have any scintillator replaced.

Of course, other problems might occur. An example is the uncertainty in the neutron background [10]. It is prudent to allow all regions of the endcap to be capable of repair, while planning on regular refurbishment of none of the active elements in the endcap. 


\section{REFERENCES}

1. D. E. Groom "Radiation Levels in SSC Detectors, Snowmass 1988, p. 711.

2. SDC Technical Design Report, SDC-92-101 (1992).

3. A. Beretvas et al., "Beam Tests of Composite Calorimeter Configurations from Reconfigurable-Stack Calorimeter", submitted to Nuc. Inst. Meth.

4. A. Beretvas et al., "Hadronic Energy Resolution and Radiation Damage", FermilabFN-582, March 1992.

5. R. Kadel, "Radiation Dose Profiles and Activation of the SDC Endcap Calorimeter", SDC-92-176, January, 1992.

6. S. Han et al., "Radiation Hardness Tests of Tile/Fiber Calorimeter Structures for SDC", Fermilab-FN-596 (1992).

7. H. Mao, private communication.

8. Calorimeter Conceptual Design Tile/Fiber Scintillator Option, Sept. 3, 1991.

9. D. Green, J. Hauptman, A. Para, "Radiation Damage, Calibration and Depth Segmentation in Calorimeters, Fermilab-FN-565, May 1991.

10. D. Green, "Neutron Radiation Damage in Comparison to EM Damage at The SSC", Fermilab-FN-597, (1992). 

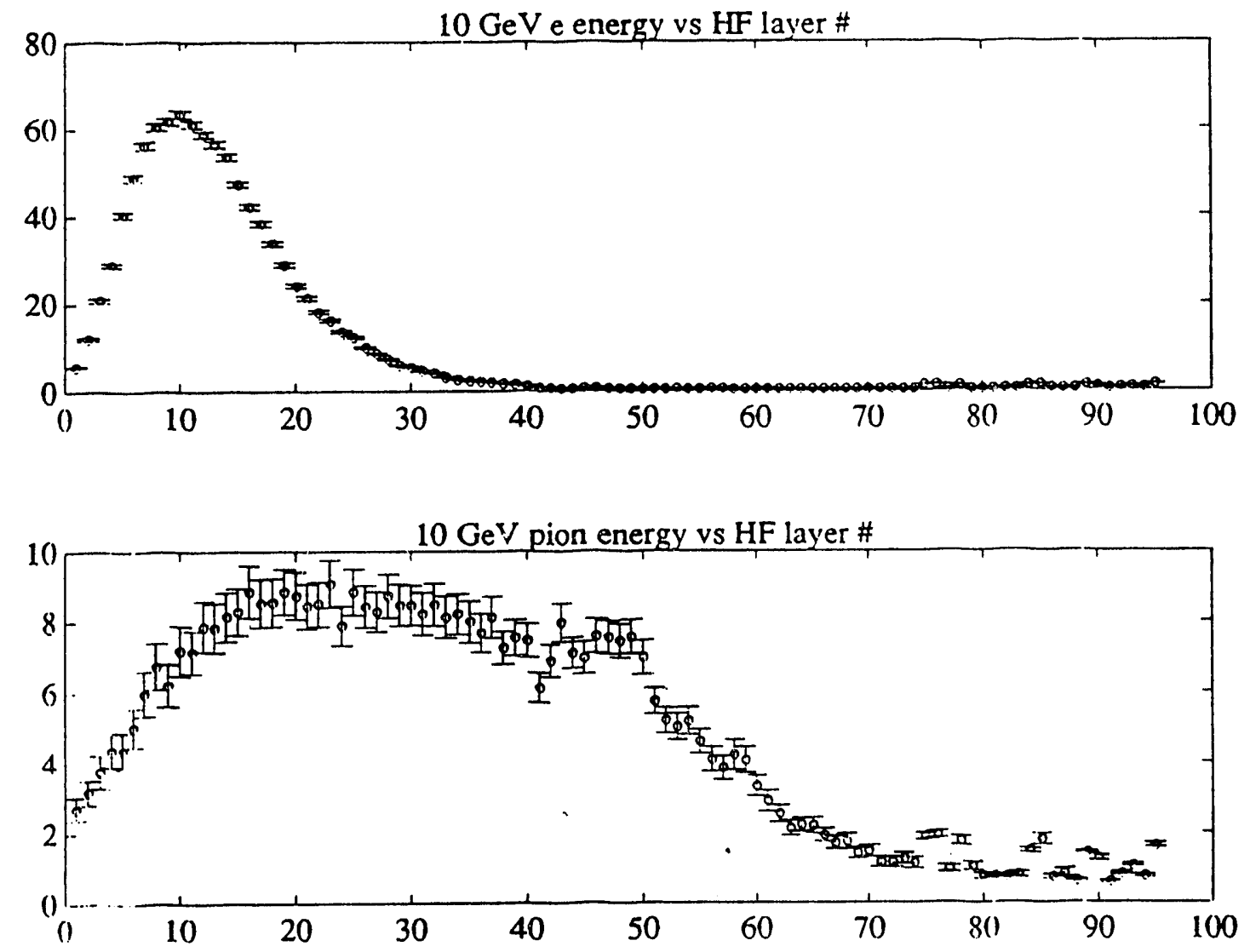

1. Longitudinal energy deposition for $10 \mathrm{GeV}$ incident particles from the "Hanging File", HF, test calorimeter. Layers 1-40 are the $\mathrm{Pb}$ EM compartment, while layers 41-95 are the Fe HAD compartment.

a. incident electrons

b. incident pions 


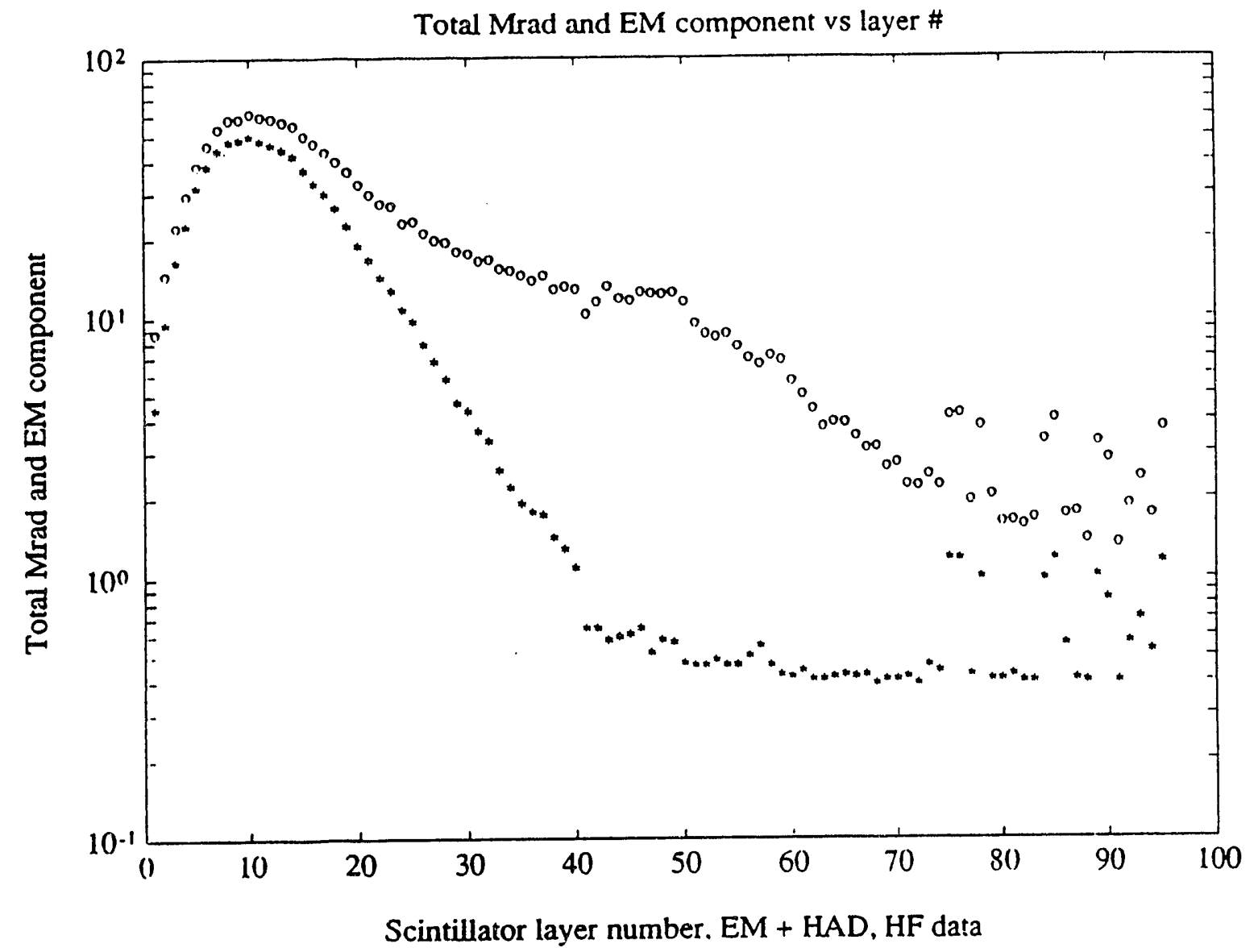

2. Longitudinal radiation dose for $10 \mathrm{GeV}$ "minbias" particles. The * points are the neutral pion dose, normalized to a peak of $50 \mathrm{Mrad}$ in the EM compartment. The o points are the total pion dose. The EM compartment is in layers 1-40; the HAD in layers 41-95. 


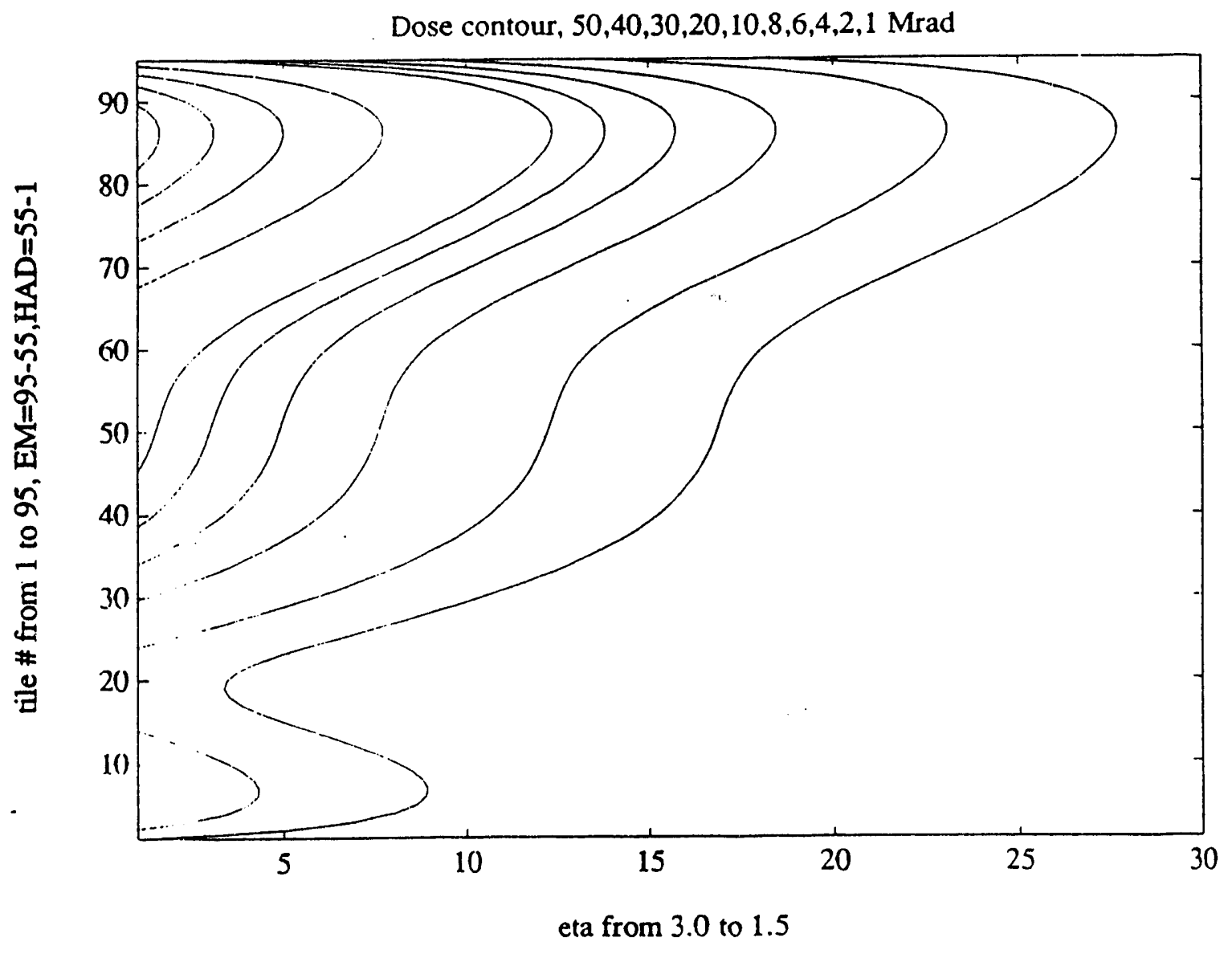

3. Contours of equal radiation dose in depth and angle. The horizontal axis is pseudorapidity from 3.0 to 1.5 , while the vertical axis is depth in layer units where $\mathrm{EM}=95-55$ and $\mathrm{HAD}=55-1$. 


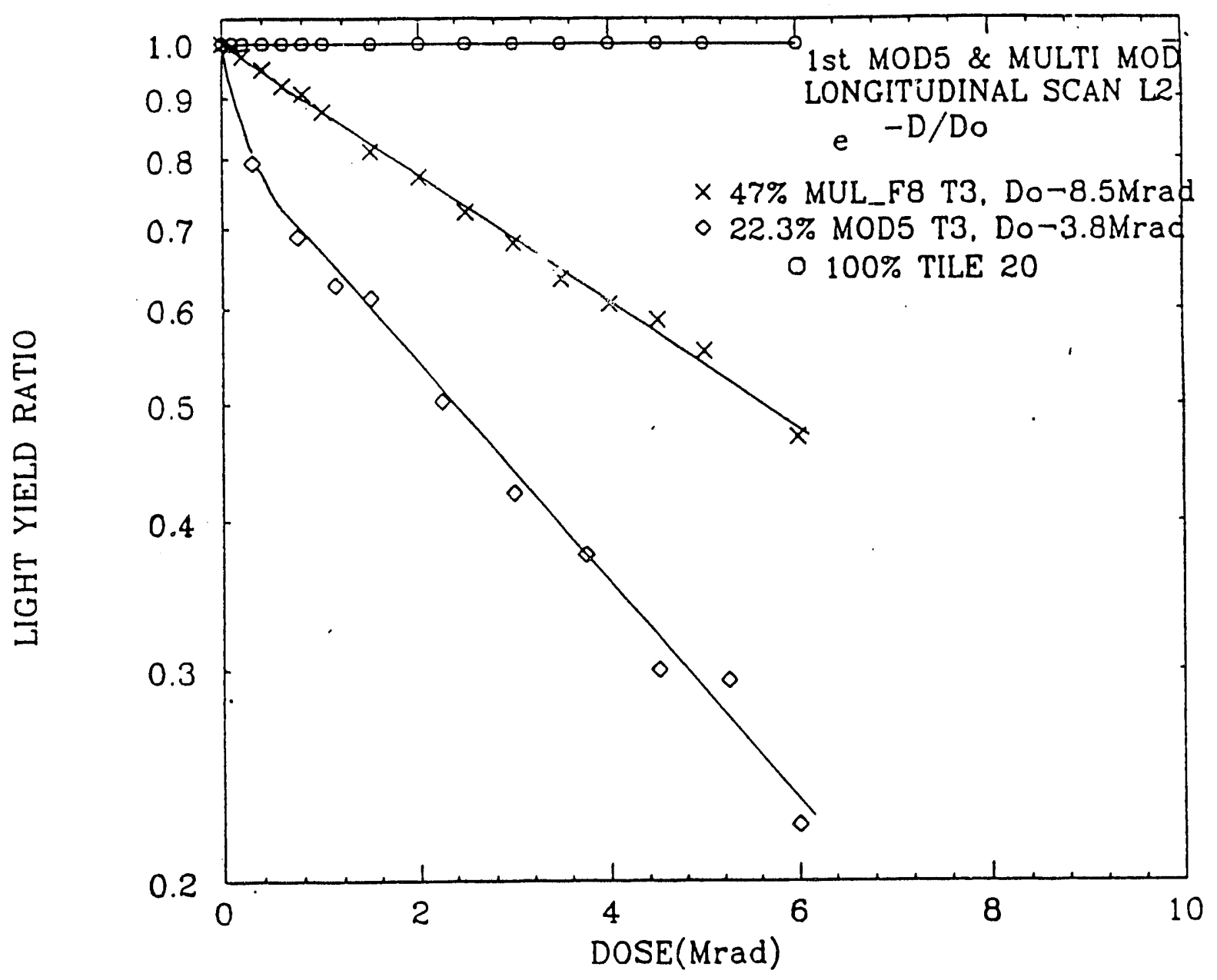

4. Relationship of dose to light output for SCSN81 + Y7 scintillator plus wave length shifter. One data set is for a standard "sigma" tile, while the other is for a "multifiber" tile containing several WLS fibers per tile. The characteristic doses, Do, are 3.8 and 8.5 Mrad respectively. 


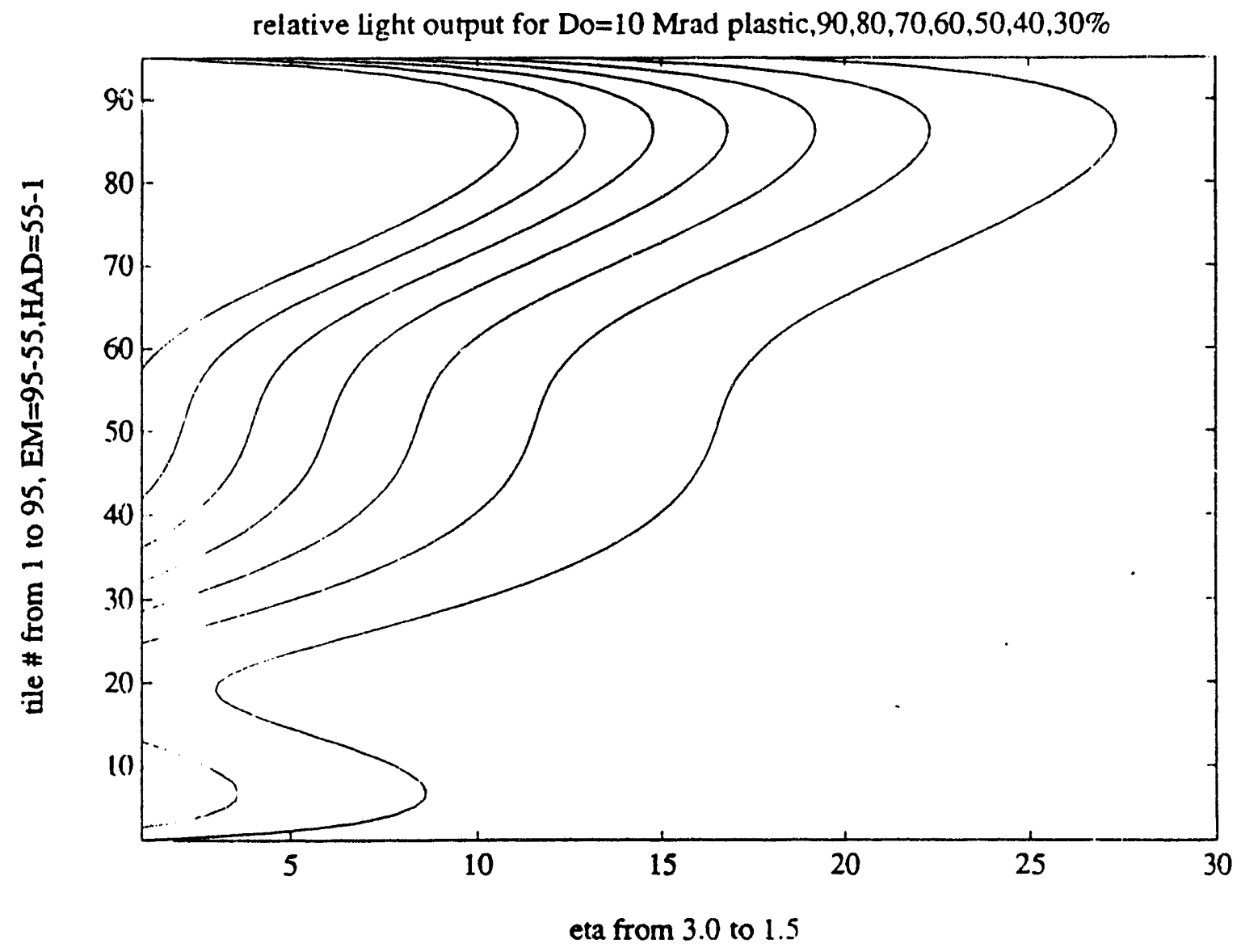

5. Contours of equal relative light output from the scintillator in depth and angle. The axes are as in Fig. 3. The characteristic dose was assumed to be Do $=10 \mathrm{Mrad}$. The relationship, $1-d=\exp (-D / D o)$ was assumed for the relationship of loss of relative light output $d$ and dose, $D$. 

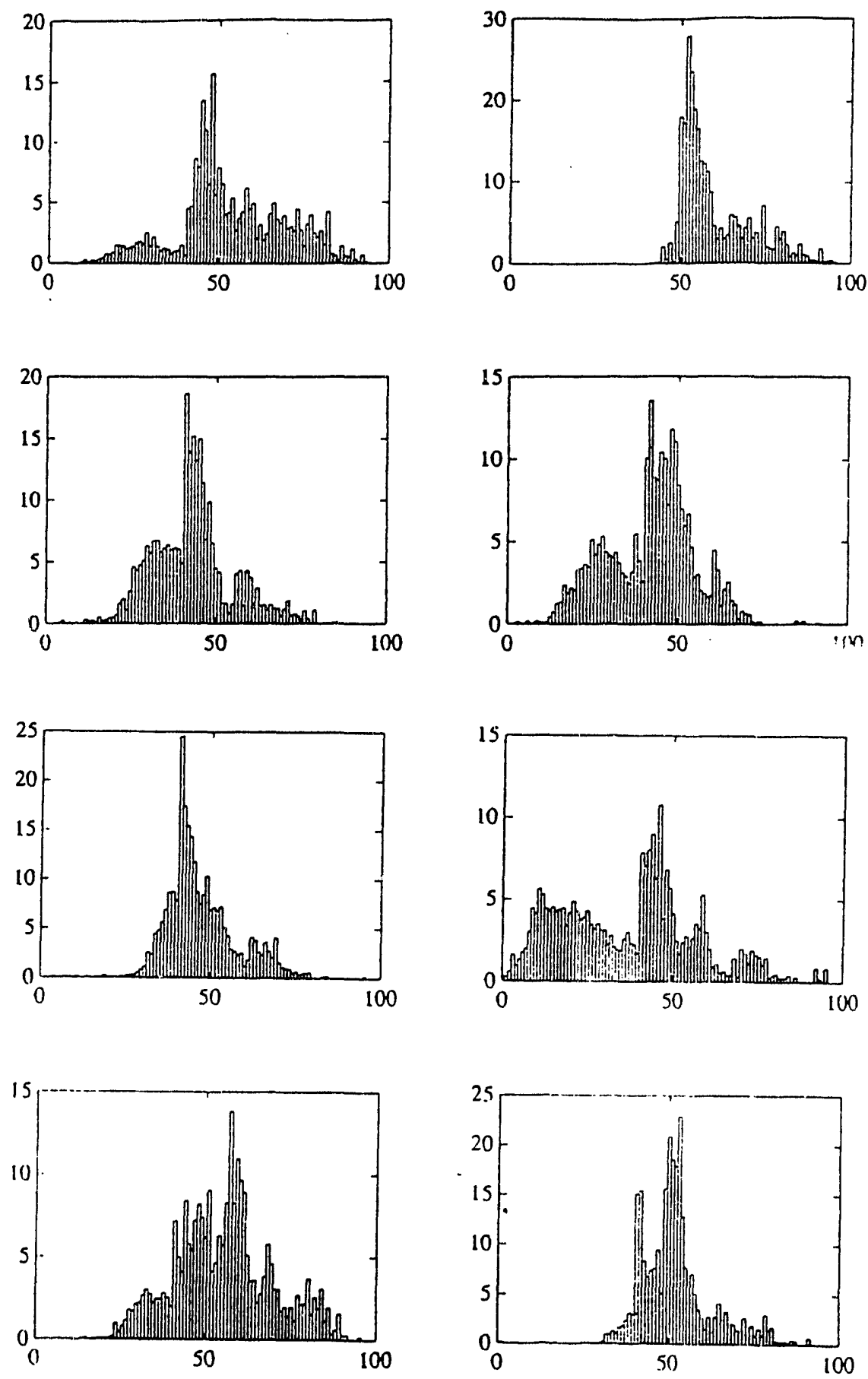

6. Event by event longitudinal energy deposition for 8 events in the HF data taken with $250 \mathrm{GeV}$ incident hadrons. Fluctuations in the conversion point and the early neutral content of the shower are evident. 

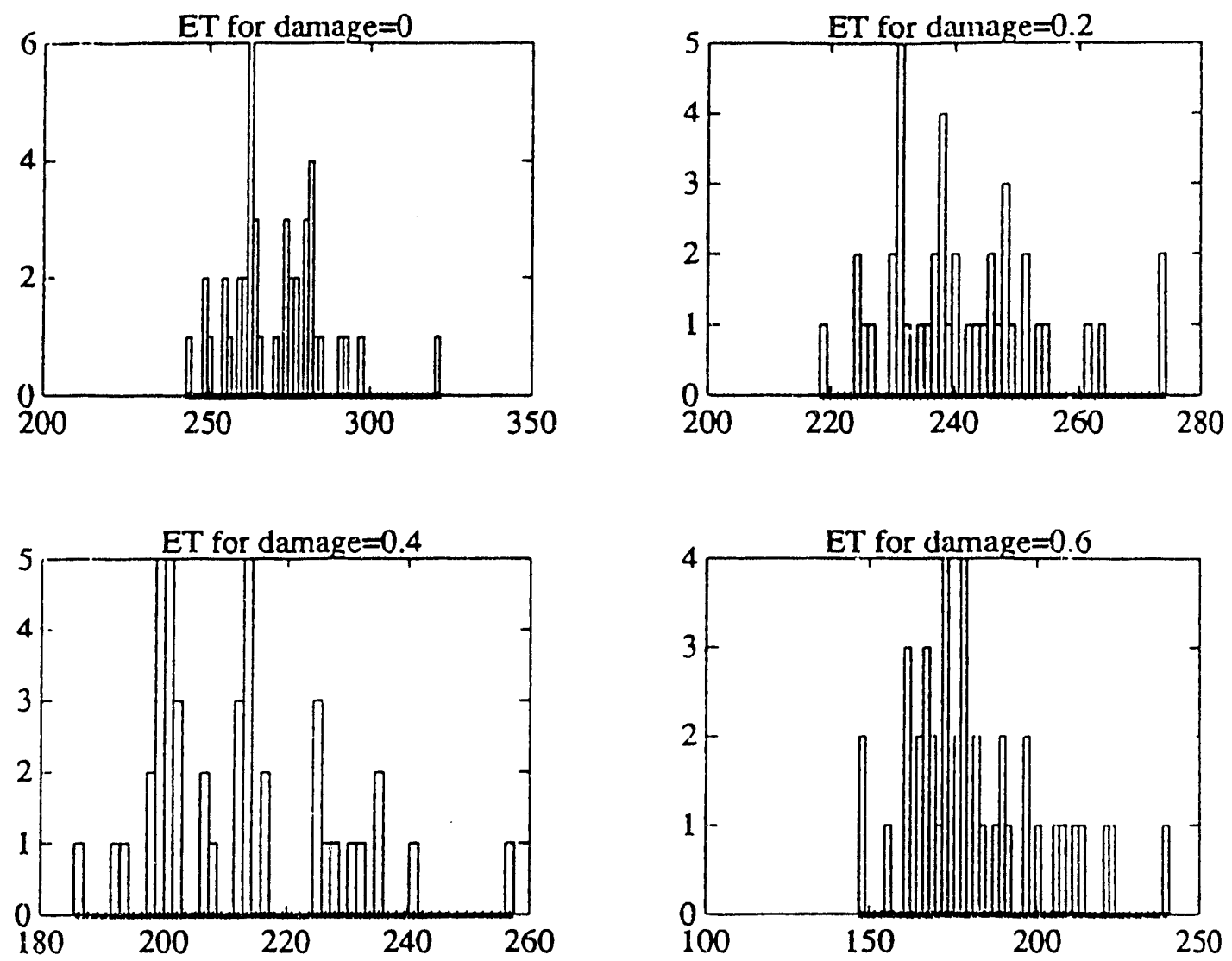

7. Energy output sum for the convolution of the radiation damage profile and the longitudinal energy deposition profile for peak damage of, $d=0.0,0.2,0.4$, and 0.6 . 

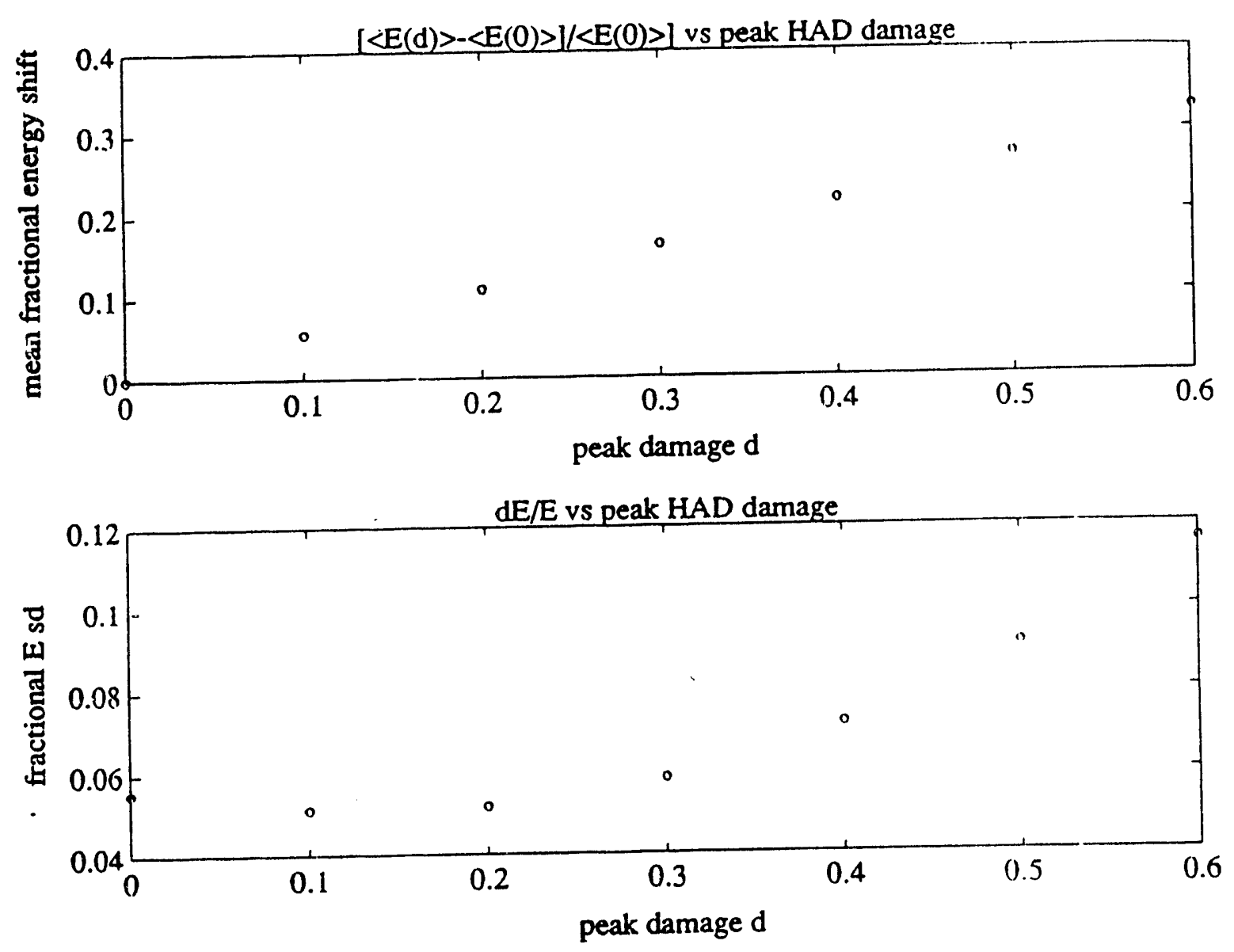

8. Energy output sum for the convolution of the radiation damage profile and the longitudinal energy deposition profile.

a. Fractional mean energy shift as a function of peak damage $d$.

b. Fracticnal energy resolution as a function of peak damage $d$. 

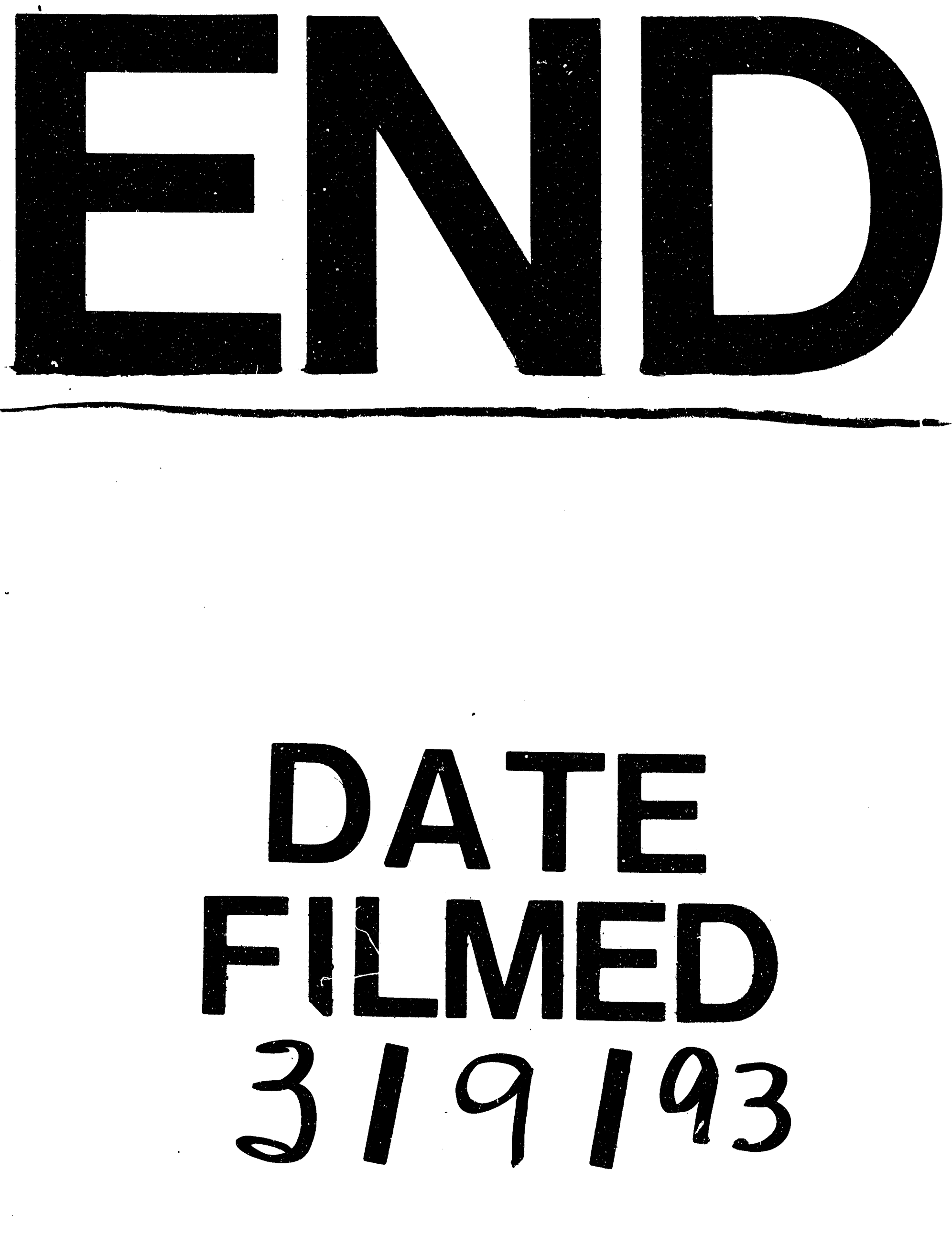
